\title{
Low-frequency scattering from submerged bubble clouds
}

\author{
Ronald A. Roya) \\ Applied Physics Laboratory, University of Washington, 1013 NE 40th Street, Seattle, Washington 98105 \\ William Carey') \\ Defense Advanced Research Projects Agency, 79 Whippoorwill Road, Old Lyme, Connecticut 06371
}

\begin{abstract}
Michael Nicholas, Jeffrey Schindall, and Lawrence A. Crum
National Center for Physical Acoustics, University of Mississippi, Coliseum Drive, University, Mississippi 38677
\end{abstract}

(Received 18 February 1992; accepted for publication 15 July 1992)

\begin{abstract}
Preliminary results are presented from a recent experiment carried out at the NUWC Seneca Lake test facility and designed to investigate the scattering properties of bubble clouds produced in fresh water in the absence of boundaries and under known propagation conditions. The test range consisted of both conventional and parametric sources, conventional receivers, and a transient bubbler that was submerged to $91 \mathrm{~m}$. The cloud possessed an elongated teardrop shape (length $\approx 1.4 \mathrm{~m}$, diameter $\approx 0.5 \mathrm{~m}$ ) with a void fraction of $0.25 \%$ and a mean bubble radius of $1.5 \mathrm{~mm}$, which corresponds to a single-bubble resonance frequency of $6.6 \mathrm{kHz}$ at depth. Backscatter target strengths were measured for frequencies ranging from $300 \mathrm{~Hz}$ to $14 \mathrm{kHz}$. These measurements revealed high target strengths (up to $+1 \mathrm{~dB}$ ) and distinctive peaks in the spectrum at $450 \mathrm{~Hz}$, and $1.3,3.0,6.5$, and $11 \mathrm{kHz}$. The low-frequency results are consistent with calculations (based on the theory of collective bubble oscillations) of the resonance frequency and target strength of a comparably sized, spherical bubble cloud. Certain features of the high-frequency backscatter data are consistent with single-bubble resonance scattering. These preliminary results are consistent with the notion that, at low frequencies, the scattering characteristics of a bubble cloud are determined, not by the bubble sizes and number density, but rather by the free-gas volume fraction and the length scales of the cloud proper.
\end{abstract}

PACS numbers: $43.30 . \mathrm{Ft}, 43.30 . \mathrm{Gv}$

\section{INTAODUCTION}

Studies indicate that microbubble layers and bubble plumes are produced when waves break, and are convected to depth by Langmuir circulation. ${ }^{1,2} \mathrm{~A}$ fundamental question to be answered is what role do microbubble layers and plumes play in the near-surface production and scattering of sound in the low-to mid-frequency range $(20 \mathrm{~Hz}$ to $2 \mathrm{kHz})$. There exists ample evidence to suggest that sound scattering from the sea surface possesses a characteristic which is different from that expected by Bragg scattering from gravity waves. ${ }^{3}$ It is our contention that breaking waves results in bubble clouds or plumes that can efficiently scatter sound. Indeed, recently published works ${ }^{4,5}$ indicate a significant role for bubble plumes in surface backscatter.

At issue is the scattering cross section for a bubble plume. Is it determined by the bubble sizes and number density or is it related to the free-gas volume fraction and the length scales for the cloud proper? Prosperettiti,7 and Carey et al. ${ }^{8,9}$ maintain that low-frequency sound is scattered primarily by the bubble plume via a process in which the bubbles pulsate collectively. In such a case, a damped resonant oscillation can result in which the eigenfrequency is determined by the phase speed within the "effective" medium (typically less than $100 \mathrm{~m} / \mathrm{s}$ ) and the characteristic length scale of the plume. Ample evidence of this phenomenon has

\footnotetext{
a) Author to whom correspondence should be sent.

b) Work performed while at NUWC, New London, CT 06320.
}

been obtained from the study of steady-state bubble columns ${ }^{10}$ and bubble screens. ${ }^{11}$

This Letter reports preliminary results from a experiment designed to measure the frequency-dependent backscattering from a bubble cloud in fresh water in the absence of boundaries and under known propagation conditions. In this effort we did not set out to duplicate "realistic" saltwater clouds, but rather to obtain data to test and validate low-frequency scattering theory in order that ocean experiments could be more effectively conceived and designed. A more complete study is currently underway.

\section{DESCRIPTION OF THE EXPERIMENT}

The Seneca Lake, NY facility consists of two moored barges in a water depth of $130 \mathrm{~m}$, with the smaller barge $(10.7 \mathrm{~m} \times 42.7 \mathrm{~m})$ serving as the platform for our test range. Equipment was deployed with the use of davits, a cable meter, and the edge of the barge, with the resulting vertical geometry shown in Fig. 1. Transmitters and receivers were oriented collinearly, with the axis of the range intersecting the path of a rising bubble cloud. The backscatter target strength for the cloud was determined over frequencies ranging from $300 \mathrm{~Hz}$ to $14 \mathrm{kHz}$ using both conventional and parametric sources. The parametric source was positioned at a depth of $61.0 \mathrm{~m}$ and driven with a $22-\mathrm{kHz}$ carrier signal that was up and down shifted by $1 / 2$ the desired difference 
Lake Surface

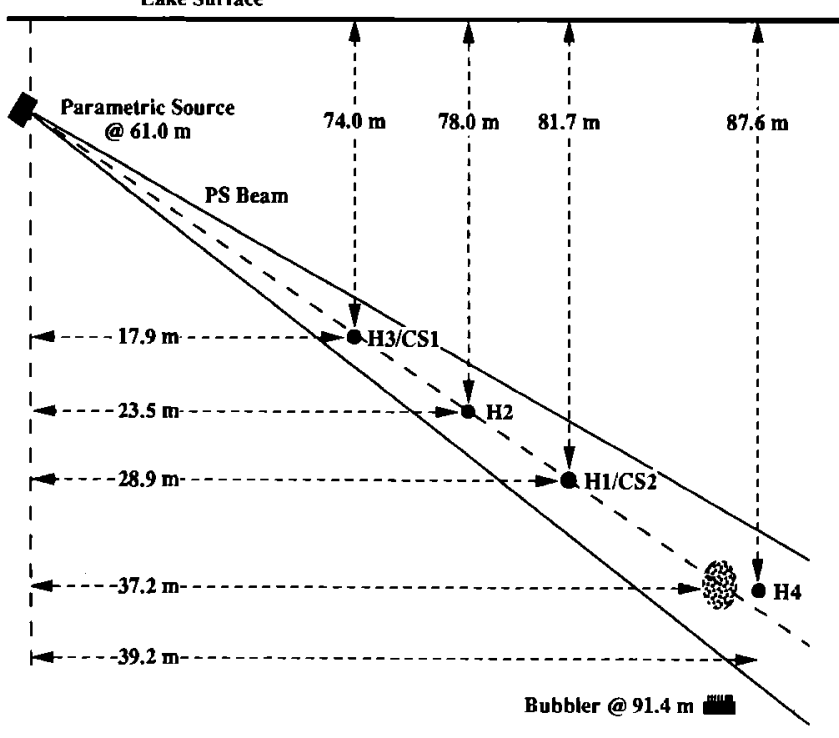

FIG. 1. Layout of the test range.

frequency, time-gated, and delivered to a $20-\mathrm{kW}$ drive amplifier. Over the course of our measurements, the repetition frequency was fixed at either 1 or $2 \mathrm{~Hz}$ and the pulse lengths were made to vary from 5 to $15 \mathrm{~ms}$. Calibrations of both the frequency-dependent source level and beamwidth were performed in situ and were nominally $177 \mathrm{~dB}$ ( re: $1 \mu \mathrm{Pa}$ at $1 \mathrm{~m}$ ) and $8.0^{\circ}$, respectively (at $1.0 \mathrm{kHz}$ ).

The parametric source offered the advantage of low-frequency directionality. However, bubbly liquids are highly nonlinear and there is therefore a possibility of an "apparent" elevation in the measured target strength due to parametric excitation. In response to this, three conventional sources were also utilized. In the $500-\mathrm{Hz}$ to $1.5-\mathrm{kHz}$ range, we employed a Honeywell HX-29 (CS1 in Fig. 1). At 3 and 5 $\mathrm{kHz}$, we used an ITC 6-in. spherical ball transmitter (CS2). At $5 \mathrm{kHz}$ and above, we drove the parametric array with conventional (i.e., single-frequency) tone bursts. We ran experiments 87.6-m depth to minimize reverberation and multipath propagation.

In addition to these sources, the test range consisted of four ITC 6-in. spherical balls, acting as receivers and situated as indicated in Fig. $1(\mathrm{H} 1-\mathrm{H} 4)$. The outputs of these hydrophones were low-pass filtered, $100-\mathrm{Hz}$ high-pass filtered, preamplified and recorded. Sound velocities were measured daily. The near-surface sound speed profile (less than 8-m depth) was variable as one might expect. At depths $>70 \mathrm{~m}$ isovelocity conditions prevailed, with a sound speed of $1421.7 \mathrm{~m} / \mathrm{s}$ at $70.0 \mathrm{~m}$ and $1420.8 \mathrm{~m} / \mathrm{s}$ at $91.4 \mathrm{~m}$. For all the calculations presented herein, a sound speed of $1421.5 \mathrm{~m} / \mathrm{s}$ was used. The range was calibrated by using the $22-\mathrm{kHz}$ primary from the parametric source to measure the time-offlight differences between hydrophones and thus verify alignment and slant ranges. We then inserted a 1.12-m-diam, hollow, steel sphere in order to test our ability to measure target strength. The target was positioned at the expected location of the bubble cloud as it crosses the axis of the range. Using the standard definition of target strength ${ }^{12}$ and assuming a perfectly reflecting target, this sphere possessed a theo- retical TS of $-11.1 \mathrm{~dB}$ at $5 \mathrm{kHz}$. Our measured TS was $-12.3 \mathrm{~dB}$.

Repeatable bubble clouds were produced using a pressurized steel enclosure submerged to $91.4 \mathrm{~m}$ and vented to the lake via an array of hypodermic needles connected to a bank of solenoid valves; the air pressure and solenoid-valve operation were controlled from the surface. The array contained forty-eight 22-gauge hypodermic needles arranged in three concentric rings of $6.25-, 12.50$-, and $18.75-\mathrm{cm}$ radii. $\mathbf{A}$ differential pressure transducer, mass-flow meter, and underwater video camera enabled us to monitor the overpressure, the total volume of air expelled into a given cloud, and the characteristic dimensions of the cloud. A 3.5-s burst of air at an overpressure of 10 psi resulted in a roughly cylindrically shaped cloud (length $\approx 1.4 \mathrm{~m}$, diameter $\approx 0.5 \mathrm{~m}$ ) with a void fraction of $0.25 \%$.

The bubble size distribution is a matter of critical importance, for it helps to distinguish single-bubble resonance scattering from multiple-bubble collective effects. By rigging the camera $\approx 2 \mathrm{~cm}$ from a needletip we obtained video images of bubbles from which we estimated the in situ distribution of bubble sizes at depth (see Fig. 2). This distribution peaks at about 1.55 -mm radius, which corresponds to a reasonance frequency of $\approx 6.6 \mathrm{kHz}$ at a depth of $87.6 \mathrm{~m}$. Even the largest bubbles ( $<3 \mathrm{~mm}$ ) possessed resonance frequencies of order $3.4 \mathrm{kHz}$. For frequencies less than $\approx 2 \mathrm{kHz}$, single-bubble resonance scattering can be neglected.

\section{EXPERIMENTAL PROCEDURE}

Target strength measurements were performed in the following manner. Once the range was aligned and calibrated and the source frequency and pulse duration determined, the solenoids were activated for $3.5 \mathrm{~s}$, resulting in a rising bubble cloud. The cloud rise velocity was approximately 0.3 $\mathrm{m} / \mathrm{s}$, which yielded a time-varying backscattered echo level. The echo level evolved as the cloud traversed the beam, reaching a maximum level as the cloud crossed the beam axis. We monitored the evolution of the echo level by digitizing the echo return, storing subsequent returns in memory, and displaying a time-compressed view of the sequence of echoes produced by the passage of a single cloud, as shown in Fig. 3. The echoes were range-gated to remove the incident

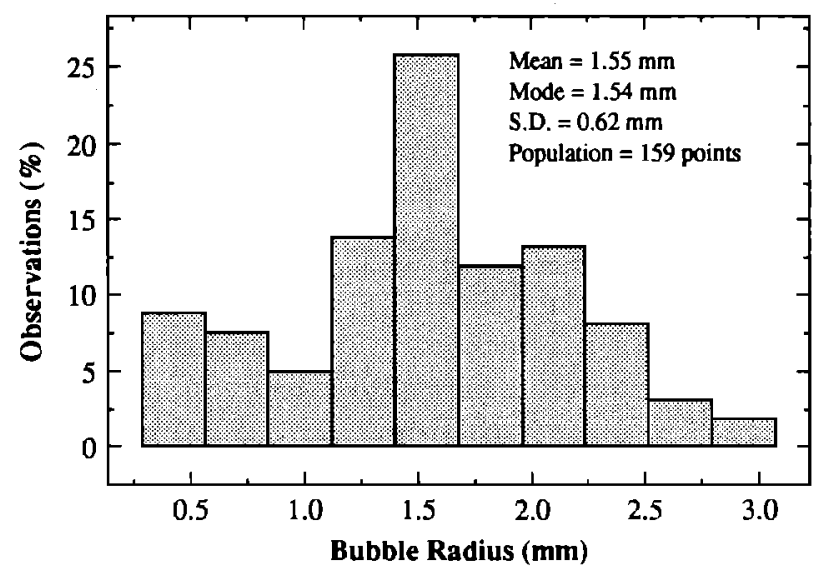

FIG. 2. Bubble-size distribution measured at a depth of $91.4 \mathrm{~m}$. 


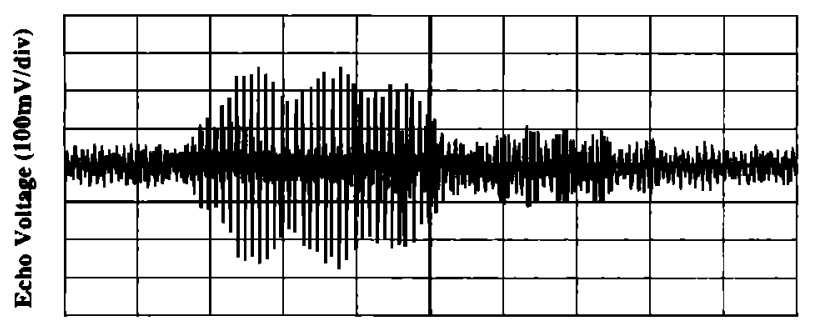

TIme Elapsed from the Generation of the Bubble Cloud (5sec/div)

FIG. 3. Typical scattering results from hydrophone $\mathrm{H} 1$, obtained with the parametric source operating at a $3.0-\mathrm{kHz}$ difference frequency. This timecompressed view of 100 echo returns serves to illustrate the evolution of the echo level as the cloud rises through the source beam. The target strength for this cloud was $-1.2 \mathrm{~dB}$

wave and to minimize reverberation. Note that the first maximum in Fig. 3 occurs at approximately the 13-s mark, which corresponds to the amount of time it takes the cloud to rise $3.8 \mathrm{~m}$ into the axis of the beam. The secondary echoes may be the consequence of angle-dependent scattering and are a subject of ongoing study.

The echo corresponding to the peak response was chosen for the determination of target strength, which we computed by measuring the peak voltage of the main pulse (MV) and the echo (EV) and inserting these quantities, along with the transmission loss factor (TLF), into the following expression for the target strength (TS):

$$
\begin{aligned}
\mathrm{TS}= & 20 \log [\mathrm{EV} / \mathrm{MV}]-\mathrm{TLF} ; \\
& \mathrm{TLF}=\mathrm{TL}_{s r}-\mathrm{TL}_{s t}-\mathrm{TL}_{t r},
\end{aligned}
$$

where the TLF accounts for the various transmission losses assuming spherical spreading of the propagating waves. The target strengths presented below consist of either single measurements or the mean values calculated from 2 to 3 repeated measurements. Error bars cannot be rigorously defined at this point due to the lack of statistical information regarding the precision of the calculated mean values; however, an ad hoc comparison of repeated measurements indicate fluctuations in the measured target strengths which ranged from \pm 0.5 to $\pm 4 \mathrm{~dB}$.

\section{EXPERIMENTAL RESULTS}

Target strengths in the mid- to high-frequency range are plotted in Fig. 4. The measured target strengths are quite large-recall that a 1-m-diam perfectly reflecting sphere has a target strength of $-12 \mathrm{~dB}$ at $5 \mathrm{kHz}$. There is evidence of resonance scattering peaks at $1.3,3.0,6.5$, and possibly 11 $\mathrm{kHz}$. From the bubble-size distribution, one can argue that the peak in the TS curve at about $6.5 \mathrm{kHz}$ is due to singlebubble resonance scattering. However, the distribution does little to account for the other peaks, which are a focus of ongoing study.

Shown in Fig. 5 are the measured low-frequency target strengths; the parametric source data were obtained at two distinct tests run during July and September of 1991 . Once again the target strengths are remarkably high; at $450 \mathrm{~Hz}$, the measured TS is about $10 \mathrm{~dB}$ greater that one would expect from a $1-\mathrm{m}$-diam perfectly reflecting sphere. There are

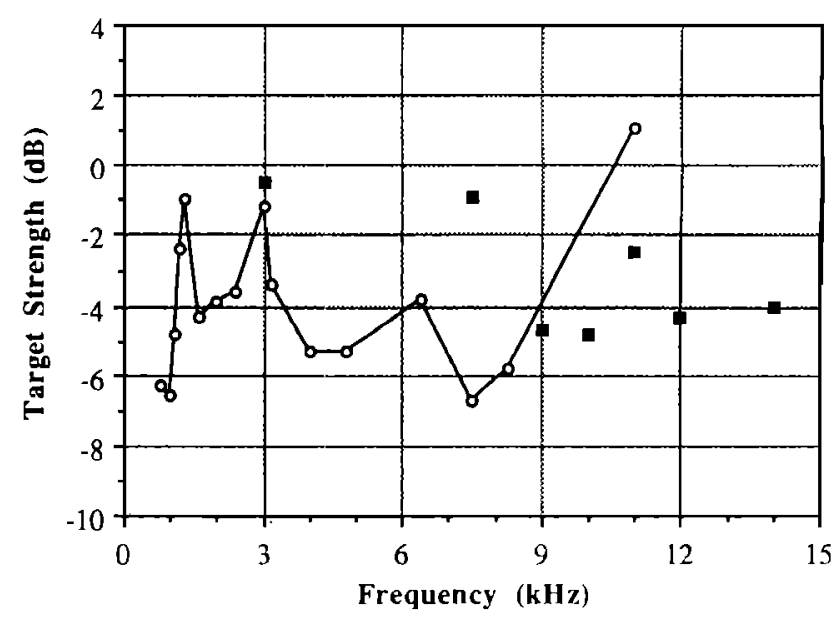

FIG. 4. Backscatter target strengths measured in the mid- to high-frequency range. The solid squares were conventional-source measurements obtained by driving the parametric source with a single primary. The open circles were obtained using the parametric source. The solid line is added to help illustrate the trends.

apparent resonance peaks at $1.3 \mathrm{kHz}$ and $450 \mathrm{~Hz}$. For these peaks to be the result of single-bubble resonance scattering the bubbles would have to possess radii of $7.9 \mathrm{~mm}$ and 2.3 $\mathrm{cm}$, respectively, which is highly unlikely.

It is probable that these peaks are the result of resonance scattering due to collective oscillations of the bubble cloud. The fundamental resonance for a spherical cloud is given approximately by ${ }^{9}$

$$
f_{0} \approx \frac{1}{2 \pi a}\left(\frac{3 \gamma P_{0}}{\chi(1-\chi) \rho}\right)^{1 / 2}
$$

where $\gamma, P_{0}, \rho$, and $a$ are, respectively, the polytropic exponent, the ambient pressure, the water density, and the effective radius of the cloud treated as a sphere. The void fraction $\chi$ is the ratio of the volume of free gas within the cloud to the total volume of the cloud. [Equation (2) holds for $0.002<\chi<0.94$.] A cloud of void fraction 0.0025 and "effective" radius $0.5 \mathrm{~m}(1 / 2$ the average of the cloud diameter and the cloud length) possesses a calculated resonance fre-

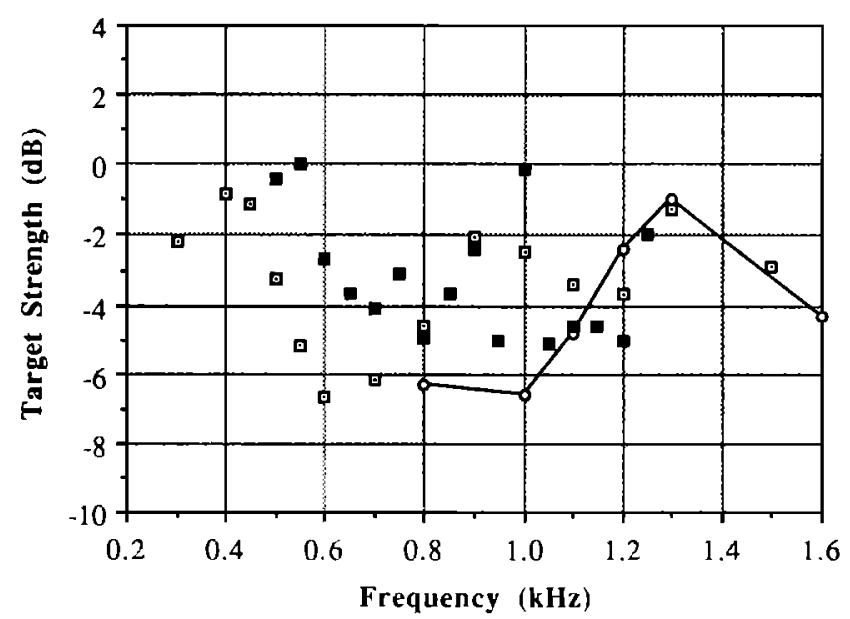

FIG. 5. Backscatter target strength measured in the low- to mid-frequency range. The solid squares were obtained using the HX-29 conventional source positioned as shown in Fig. 1. The open circles and dotted squares were the results of parametric-source measurements run in July 1991 and October 1991, respectively. 
quency of approximately $405 \mathrm{~Hz}$. Moreover, the target strength is given by ${ }^{12}$

$$
\text { TS }=10 \log \left[I_{s} / I_{i}\right]_{r=1 \mathrm{~m}},
$$

where $I_{s}$ and $I_{i}$ are the scattered and incident intensities, respectively. For the bubble cloud oscillating collectively, this is given approximately by ${ }^{9}$

$$
\begin{aligned}
\frac{I_{s}}{I_{i}} \approx & \frac{z_{0}{ }^{6}}{9(k r)^{2}} \frac{\left\{1-\left(x / x^{\prime}\right)\right\}^{2}}{\left[1-\left(x / x^{\prime}\right) z_{0}{ }^{2} / 3\right]^{2}+\left[\left(x / x^{\prime}\right) z_{0}{ }^{3} / 3\right]^{2}}, \\
& \quad r=1 \mathrm{~m},
\end{aligned}
$$

where $z_{0}=k a, x=\rho c^{2}$, and $x^{\prime}=\rho_{m} c_{m}^{2}$, where the subscript $m$ denotes the effective properties of the air/water mixture. (We use the definition of the void fraction to obtain $\rho_{m}$ and Wood's result ${ }^{13}$ to determine $c_{m}$.) Since Eq. (4) does not account for thermal damping, this result is only accurate provided the damping decrement is of order $3 \times 10^{-3}$ or less. For a 0.5 -m-radius cloud with a void fraction of 0.0025 , we calculate a target strength of approximately $-6 \mathrm{~dB}$ at 405 $\mathrm{Hz}$. These values for the resonance frequency and target strength of the cloud exhibit qualitative agreement with our measurements in the $400-500 \mathrm{~Hz}$ range.

A comparison between the low-frequency measurements obtained with the parametric and conventional sources indicate reasonably good agreement. Although reverberation made it difficult to obtain conventional source data for frequencies less than $1 \mathrm{kHz}$, data obtained in the $500-\mathrm{Hz}$ to $3-\mathrm{kHz}$ range using the parametric source, the $\mathrm{HX}$ 29, and the ITC 6-in. sphere were comparable. However, parametric and conventional source measurements at 1,7 , and above $9 \mathrm{kHz}$ differed by as much as $5 \mathrm{~dB}$. (Our confidence in the $1-\mathrm{kHz}$ conventional source measurement is low, due to the large spread in the data at this frequency. An analysis of all the data obtained at this frequency has yet to be performed.) It appears that enhanced parametric excitation did not appreciably bias the target strength measurements for frequencies below about $9 \mathrm{kHz}$.

\section{CONCLUSIONS}

Measurements of backscatter TS versus frequency have been made using conventional and parametric sources. The results using these two types of sources were found to be comparable for frequencies ranging from $500 \mathrm{~Hz}$ to $3 \mathrm{kHz}$ (the $1-\mathrm{kHz}$ data not withstanding). Backscatter TS were quite high, exceeding that of a comparably sized, perfectly reflecting sphere by as much as $15 \mathrm{~dB}$. Certain features of the high-frequency backscatter data are consistent with singlebubble resonance scattering while measurements at the low frequencies $(<1 \mathrm{kHz})$ appear to be governed by the collec- tive oscillations of the cloud. It is evident that several features of the backscatter spectra require additional analysis.

In summary, we conclude that our measured target strengths reflect both single and collective bubble behavior, with the latter playing the more prominent role at the lower frequencies.

\section{ACKNOWLEDGMENTS}

The authors would like to thank Martin Wilson of the Univ. of Connecticut as well as Joe Burch, Paul Dakin, and Ali Mehrabi of NCPA for their lending their expertise to this effort. We would especially like to thank William Konrad of NUWC and William Marshall of BBN for their expert assistance with the parametric and conventional sources, and Andrea Prosperetti of Johns Hopkins Univ. for his insight and advice. Finally, we acknowledge the financial support of Marshall Orr, ONR; Ken Lima, NUWC IR; as well as AEAS, ONT, and DARPA.

'S. A. Thorpe, "On the clouds of bubbles formed by breaking wind-waves in deep water and their role in air-sea gas transfer," Philos. Trans. R. Soc. London Ser. A 304, 155-185 (1982).

${ }^{2}$ E. C. Monahan and M. Lu, "Acoustically relevant bubble assemblages and their dependence on meteorological parameters," IEEE J. Ocean Eng. 15(4), 340-349 (1990).

${ }^{3}$ S. McDaniel, "High-frequency sea surface scattering: Recent progress," J. Acoust. Soc. Am. Suppl. 184, S121 (1988).

${ }^{4}$ B. E. McDonald, "Echoes from vertically striated subresonant bubble clouds: A model for ocean surface reverberation," J. Acoust. Soc. Am. 89, $617-622$ (1991).

${ }^{5} \mathrm{~F}$. Henyey, "Acoustic scattering from ocean microbubble plumes in the $100 \mathrm{~Hz}$ to $2 \mathrm{kHz}$ region," J. Acoust. Soc. Am. 90, 399-405 (1991).

'A. Prosperetti, "Bubble related ambient noise in the ocean," J. Acoust. Soc. Am. 84, 1042-1054 (1988).

'A. Prosperetti, "Bubble dynamics in ocean ambient noise," in Sea Surface Sound, edited by B. R. Kerman (Kulwer Academic, Boston, MA, 1988), pp. 151-171.

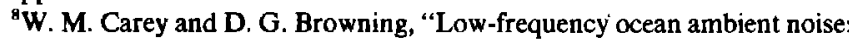
Measurements and theory," in Ref. 7, pp. 361-376.

${ }^{9}$ W. M. Carey, J. W. Fitzgerald, and D. G. Browning, "Low-frequency noise from breaking waves," in Natural Physical Sources of Undenwater Sound, edited by B. R. Kerman (Kluwer Academic, Dordrecht, Netherlands, 1990). [Also: NUSC Tech. Rep. 8783, 5 Oct. 1990, avail. DTIC \#AD227969.]

${ }^{10} \mathrm{~S}$. W. Yoon, L. A. Crum, A. Prosperetti, and N. Q. Lu, "An investigation of the collective oscillations of a bubble cloud," J. Acoust. Soc. Am. 89, 700-706 (1991).

"I. N. Kozhevnikova and L. Bjorno, "Transformation of signals from high to low frequency in a bubble screen," in Conference Proceedings: Ultrasonics International 1991 (Butterworth, London, 1991), pp. 591-595.

${ }^{12}$ R. J. Urick, Principles of Underwater Sound (McGraw-Hill, New York, 1975), pp. 263-278.

${ }^{13}$ A. B. Wood, A Textbook of Sound (Bell, London, 1932/1955), pp. 360364. 